\title{
Regional Variation within the Interphotoreceptor Matrix from Fovea to the Retinal Periphery
}

\author{
J. G. HOLLYFIELD, H. H. VARNER, M. E. RAYBORN \\ Houston, USA
}

\begin{abstract}
Summary
The interphotoreceptor matrix in the human retina comprises a highly organised extracellular compartment. Using fluorescent labelled peanut agglutinin and the cationic dyes Cupromeronic and Cuprolinic Blue, unique cylindrical domains surrounding cone photoreceptors can be demonstrated. These cone specific domains are relatively insoluble and are closely adherent to cone photoreceptors and to the pigment epithelium, suggesting that these structures may play a role in retinal attachment.
\end{abstract}

The interphotoreceptor matrix (IPM) which occupies the compartment bounded on one side by the retina and the other by the pigment epithelium was for many years considered only as an amorphous entity. ${ }^{1}$ More recently, studies employing peanut agglutinin probes (PNA) and the cationic dyes, Cuprolinic and Cupromeronic Blue, which are highly selective stains for sulfated proteoglycans, have extended our understanding of the organisation of this compartment. ${ }^{2,3,4,5}$ These studies clearly establish the presence of highly organised subdomains within the IPM. Each cone photoreceptor is surrounded by a highly organised cylindrical sheath beginning at the outer limiting membrane and extending to the apical surface of the pigment epithelium. In some species, particularly primates, in addition to the presence of a PNA binding component, these structures also contain chondroitin sulfate proteoglycans..$^{3,6}$

The interactions occurring between the photoreceptors, the apical surface of the pigment epithelium, the PNA-binding glycoproteins, and chondroitin sulfate proteoglycans present in the cone matrix sheaths are, as yet, unclear. However, experimental retinal detachment studies indicate that the cone matrix sheaths are strongly adherent to both the cone photoreceptor and to apical processes of the pigment epithelium, which implicates these IPM components as being involved in attaching the outer retina to the pigment epithelium. This paper will present a short review of the regional variation of cone specific domains in the human retina and discuss a possible role for those components in retinal attachment.

\section{Methods}

All of our studies on the IPM in the human retina utilise tissues obtained from the Lions Eyes of Texas Eye Bank, Houston, with postmortem times of under one hour. Fixation and staining of retinal tissues with Cuprolinic and Cupromeronic Blue have been detailed in previous studies. ${ }^{4,5}$ The epifluorescent microscopy describing regional variation in FITClabelled PNA binding to cone matrix domains has been performed on the isolated photoreceptor layer, utilising a modification of a method developed for isolating the IPM from bovine retinas ${ }^{7}$ which will be described elsewhere. $^{8}$ 

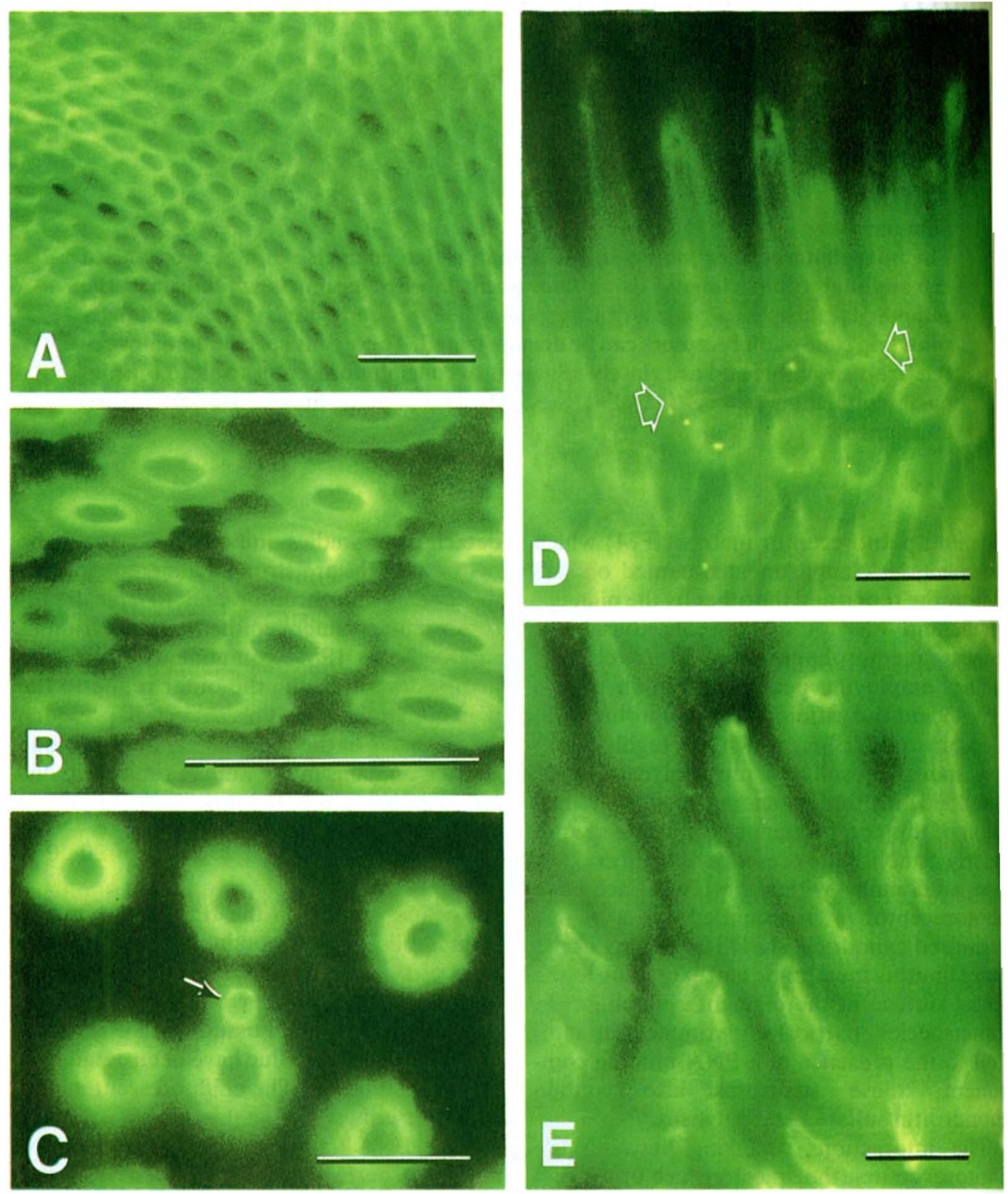


\section{PNA Staining of Cone Matrix Domains}

FITC-labelled peanut lectin is an excellent probe for demonstration of the regional variation in the structure of cone specific matrix domains at different retinal locations. In the fovea, a PNA-positive matrix fully occupies this extracellular compartment (Fig. 1A). When the isolated photoreceptor layer is viewed as a flat mount from the level of the outer limiting membrane looking in a sclerad direction, the entire PNA-positive complex has the appearance of a honeycomb with circular shaped holes extending through the PNA-positive matrix at regular intervals. A single foveal cone extends through the outer limiting membrane into the lumen of each of these sieve-like openings. In the wall of the matrix, approximately midway between the luminal margins, a slightly brighter fluorescent line is present which forms a hexagonal pattern in the matrix surrounding each luminal opening. The overall appearance of the IPM in the fovea utilising this technique is that it forms a continuous, highly organised extracellular environment completely surrounding the entire population of foveal cones.

In perifoveal locations, the individual cone matrix domains become progressively separated from each other as rod photoreceptors make their appearance. These domains have thicker walls with a smooth circular lumen and a scalloped peripheral border (Fig. 1B). Each scalloped indentation at the periphery of the PNA-positive domain is occupied by a closely apposed rod photoreceptor. In the mid-to-far periphery the individual cone matrix domains are further separated from each other as the density of rod photoreceptors increases in these locations (Fig. 1C).

When viewed from the side, the individual cone matrix domains are extremely thin and elongated at the fovea, but are more truncated and with tapering tips which were located proximal to the pigment epithelium prior to retinal isolation (Figs. 1D and 1E).

In addition to binding to the cone matrix domains in the peripheral retina, PNA labels a small population of rods as well (Figs. 1B and $1 \mathrm{C}$ ). These PNA-binding rods represented less than one per cent of rod photoreceptors and do not appear to occur in any apparent pattern. Some were adjacent to cones while others were located several rod diameters away from the nearest cone.

\section{Cuprolinic Blue Staining of Cone Matrix Domains}

Studies using the cationic copper phthalocyanin dye Cuprolinic Blue and Cupromeronic Blue using conditions for selective staining of sulfated polyanions ${ }^{9}$ have allowed the visualisation of cone matrix domains with electron

Fig. 1. (A) Flat mount preparation of the isolated outer retina from the fovea viewed with epifluorescence following staining with FITC-labelled peanut agglutinin. In Figs 1A-C the field of view is at the level of the outer nuclear layer looking outward toward the pigment epithelium. The level of focus is just beyond the outer limiting membrane, in the region of the cone matrix domains occupied by the photoreceptor inner segments. The dark holes through the FITC staining matrix represent the compartments occupied by cone photoreceptors. Note that these IPM domains in the fovea appear as a continuous, uninterrupted layer penetrated only by the cylindrical openings where cone photoreceptors extend from the outer retina toward the pigment epithelium. At several locations in the fluorescent labelled wall between the dark openings, alternating dark to light lines can be seen which may represent the interface between abutting cone matrix domains. Compare with figure 3.

(B) Cone matrix domains from perifoveal region. In this location, as rod photoreceptors appear, some of the cone matrix domains are separated from each other by rod photoreceptors, while others continue to abut directly onto other cone matrix domains. The lumen of the cone matrix domains in this region have larger diameters than those in the fovea to accommodate the larger cone inner and outer segments present in these extrafoveal locations.

(C) Cone matrix domains from peripheral retina. In this location the cone matrix sheaths are separated from each other by several rod diameters. The margin of the cone matrix domains in all retinal locations, except the fovea, have a scalloped border which are shaped by the closely opposed rod photoreceptors. One rod photoreceptor (arrow) shows intense labelling.

(D) In some locations in the flat mount preparations, the edges of the tissue curls, allowing the visualisation of the FITG labelled PNA domains along their full length. In this preparation from the perifovea, the domains are broadly expansive where they appear to originate at the outer limiting membrane (between open arrowheads) and gradually taper until they end at the tips which were broken from the pigment epithelium during the isolation.

(E) Flat mount preparation of the perifoveal retina viewed from the level of the pigment epithelium looking downward onto the tips of brightly fluorescent cone matrix sheaths. The level of focus is near the tips of the cone matrix sheath that would abut onto the pigment epithelium. Horizontal bars in each micrograph represent $5 \mu \mathrm{m}$ in length. 


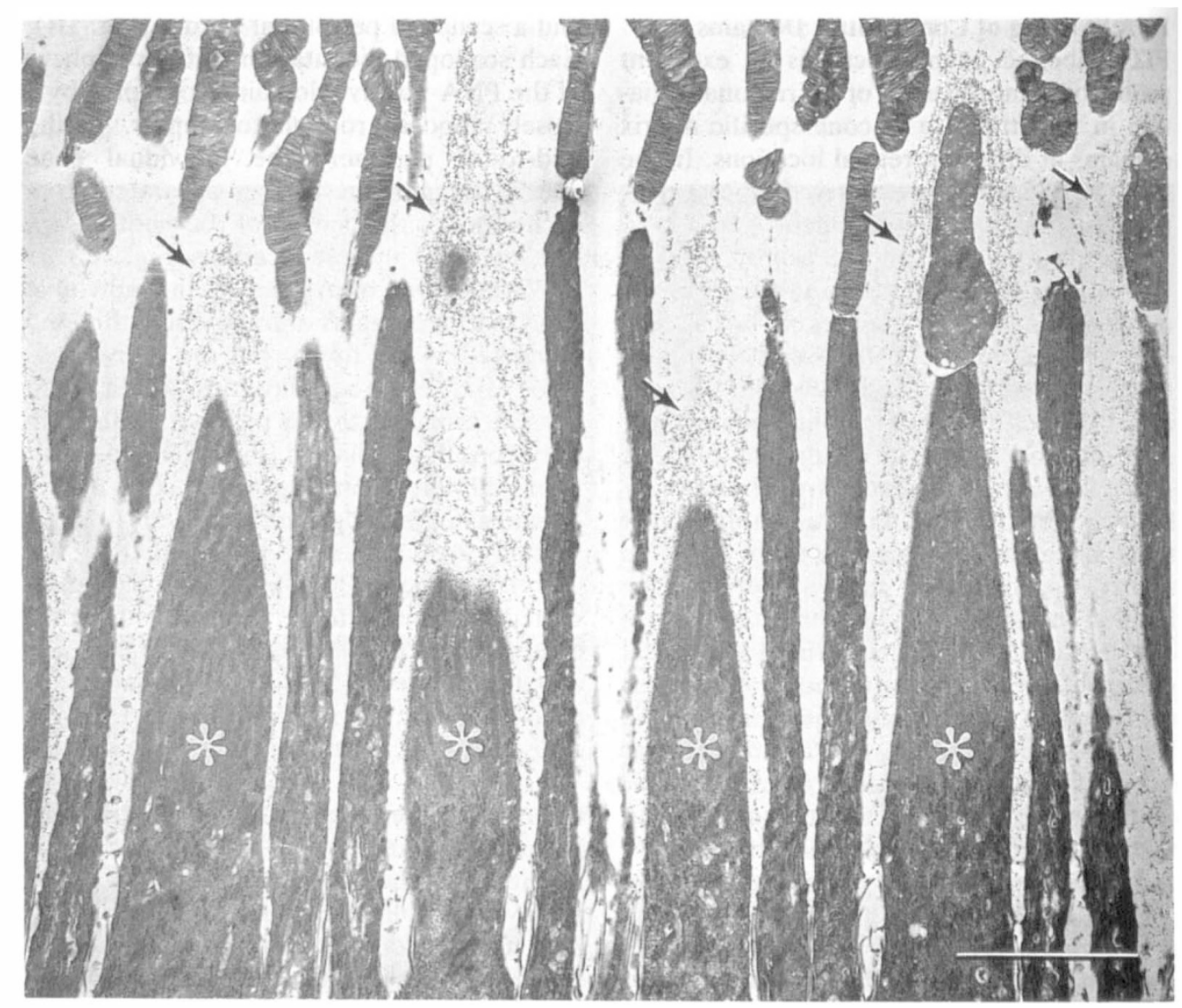

Fig. 2. Electron micrograph of perifoveal retina stained with Cupromeronic blue to demonstrate the presence of the cone matrix domains. Asterisks designate the inner segment of several cone photoreceptors and the arrows point to the fibrillar, Cupromeronic blue positive particles which comprise the cone matrix domains. Some linear profiles indicating a laminar arrangement of the unit particles are evident in this low magnification micrograph. Magnification bar in lower right of micrograph represents $5 \mu \mathrm{m}$.

microscopy. ${ }^{3,4}$ The matrix domains resolved with this method extend from the outer limiting membrane of the retina to the apical surface of the pigment epithelium. Their staining is eliminated by preincubation of retinal tissues with chondroitinase AC, suggesting that sulfated proteoglycans stained with these dyes represent chondroitin sulfate-type proteoglycans. ${ }^{3}$ When isolated free of the pigment epithelium and rinsed in saline to remove soluble IPM components prior to staining, the cone matrix domains remain associated with the cone inner and outer segments (Fig. 2) suggesting that the proteoglycan component is physically attached to the cone.

Regional variations in the sizes and shape of the unit particles stained with these dyes were also evident. In peripheral retina, the particles comprising the sheath were punctate with an average diameter of $40 \pm 12 \mathrm{~nm}$. Particles were more elongate in the sheaths surrounding foveal cones $(20 \mathrm{~nm}$ in diameter and $50-60 \mathrm{~nm}$ in length) and were closely packed with no particle free zones between individual domains (Fig. 3). In the sheaths of perifoveal cones, two sizes of particles were observed, one in the wall proximal to the lumen $(20 \mathrm{~nm}$ in diameter and $60-80 \mathrm{~nm}$ in length). and another distal to the lumen $(30-40 \mathrm{~nm}$ in diameter and $50 \mathrm{~nm}$ in length). ${ }^{4}$

The outer extension of the cone matrix sheath ends in close association with the api- 


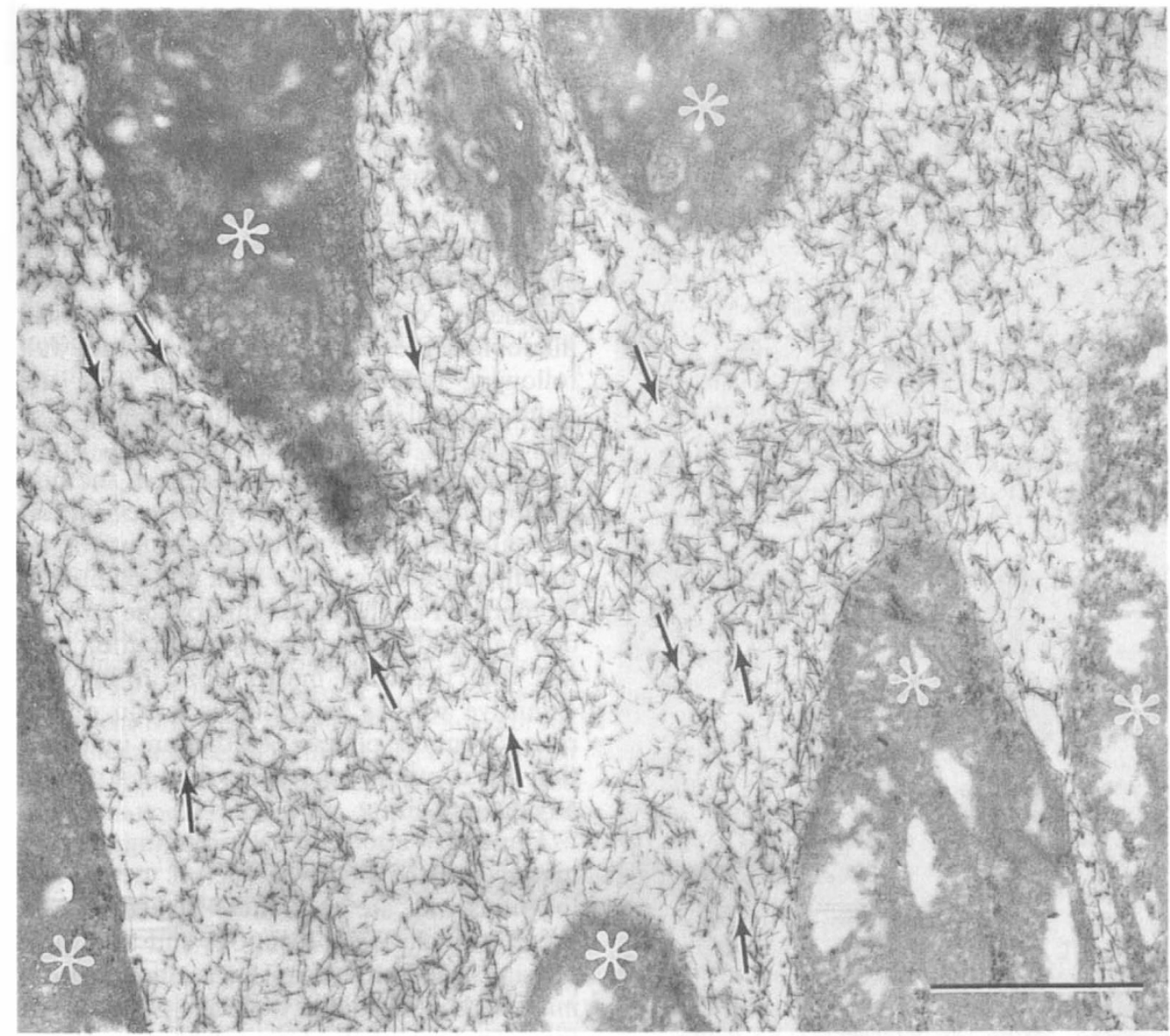

Fig. 3. Electron micrograph of Cupromeronic blue stained foveas. The tissue has been sectioned at an angle, slightly oblique to the long axis of the photoreceptors. The inner segments of the cone photoreceptors present, appear interrupted $\left(^{*}\right)$. The interphotoreceptor matrix is filled with Cupromeronic blue positive rod like filaments which show an extensive felt like arrangement. In several locations (indicated between arrows) the filaments appear to form a linear pattern which may represent the interface where the outer borders of individual cone matrix domains abut onto each other. These linear arrays of Cupromeronic blue stained particles may be equivalent to the brightly fluorescing lines present in the matrix between cones in the FITC-labelled PNA micrographs presented in Fig $1 A$ above. Magnification bar in lower right represents $1 \mu \mathrm{m}$.

cal surface of the pigment epithelium. From the apical surface, a number of short microvillae extend into the wall of the sheath and long microvillar processes extend into the sheath lumen to approach the tip of the cone outer segment. ${ }^{4}$ When retinas were isolated from the pigment epithelium and rinsed extensively prior to fixation and staining, apical microvillae were found embedded within the terminal extensions of the matrix sheaths, suggesting that the microvillae are firmly attached to the components within the sheath wall (Fig. 4).

\section{Discussion}

Observations summarised here present anatomical evidence of a component in the interphotoreceptor matrix which may serve physically to attach the retina to the pigment epithelium. This linkage is mediated by a specialised extracellular domain surrounding cone photoreceptors. To function as an attachment factor, the cone matrix sheath should meet at least two minimum criteria: it must (a) be attached to the cone photoreceptor and to the pigment epithelium and (b) be 


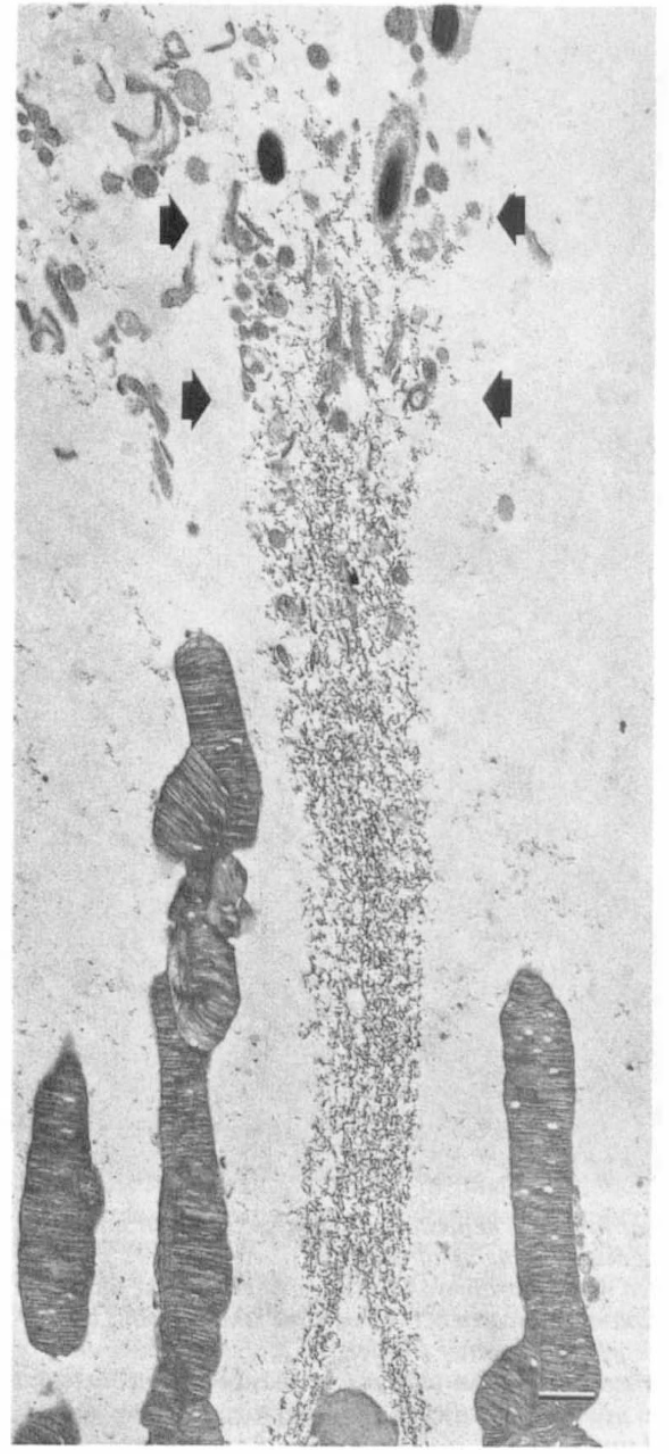

Fig. 4. Electron micrograph of peripheral retina which was isolated from the pigment epithelium and washed extensively prior to Cupromeronic blue staining. Two rod photoreceptors are on either side of a cone with the heavily stained cone matrix domain extending from the cone outer segment through the centre of the micrograph. Near the outer limit of the cone matrix domain at the top of the micrograph, broken fragments from the pigment epithelium are embedded in the cone matrix sheath wall (some of which are designated between the arrow heads). Bar in lower right represents 1 um.

relatively insoluble with a tensile strength appropriate for this bridging capability. While we have no direct evidence regarding either of these criteria, some inferences can be made based on the results of studies summarised here. We have observed that the cone matrix sheath remains associated with the cone following experimental detachment of the retina from the pigment epithelium. This indicates that a linkage exists between the cone matrix sheath and the cone. In addition, the broken fragments of pigment epithelium apical microvillae present in cone matrix sheath wall following isolation of the retina from the outer eye wall, indicates that the sheath is also adherent to the pigment epithelium. Since these microvillae were broken from the pigment epithelium during the process of experimental detachment, the tensile strength of the sheath must be greater than the combined strengths of all the apical microvillae embedded within the sheath wall. In addition, the fact that the cone matrix domain survives extensive washes following isolation of the retina from the pigment epithelium indicates that this component is an insoluble, structurally distinct entity.

The observations of PNA binding to cone matrix sheaths in foveal versus peripheral locations indicate that these structures are present at all retinal locations and that their density in various retinal locations precisely mirrors the local density of cone photoreceptors. The presence of an attachment factor associated with cone photoreceptors may in part explain the clinical observations that retinal holes in the periphery more frequently lead to retinal detachments than do holes near the macula. ${ }^{10}$ Since cone photoreceptors are sparse in peripheral retina and increase in density more proximal to the macula, the presence of an attachment factor associated only with cones would imply that the local density of cones per se may be an index of relative strength of retinal attachment in different retinal locations. Additionally, the absence of rods in the macula permitting the extensive lateral interactions between adjacent cone matrix sheaths may allow for an even stronger attachment of the retina to the pigment epithelium in this location than would be expected based on cone density alone.

While studies of components in the interphotoreceptor matrix have been underway 
for some time (See Varner $e t$ al $^{11}$ for a recent review of this area), the utilisation of PNA and the cationic dyes, Cuprolinic and Cupromeronic Blue, have only recently been utilised for an analysis of components in this extracellular compartment. A combination of biochemical and morphological techniques using these probes will be important in providing information as to the cells responsible for their synthesis, turnover and repair following detachment and reattachment of the retina to the pigment epithelium.

We thank Kathy Myers and May Lin Bell for technical and secretarial assistance. We also thank Mr. Alex Kogan for help with the photography and the Lions' Eyes of Texas Eye Bank, Houston, for their efforts in obtaining the human tissues used in this study. This study was supported by grants from the National Eye Institute, National Institutes of Health, Bethesda, MD; The RP Foundation Fighting Blindness, Inc., Baltimore, MD; The Retina Research Foundation, Houston, TX; and Research to Prevent Blindness, New York NY. Some of the instrumentation used in this study was obtained through funding by The Institute for Aid and Rehabilitation, Houston, TX. JGH is the recipient of an Alcon Research institute Award and a Research to Prevent Blindness Senior Scientific Investigators Award.

\footnotetext{
References

${ }^{1}$ Röhlich P: The interphotoreceptor matrix: Electron microscopic and histochemical observations on the vertebrate retina. Exp Eye Res 1970, 10: 80-96.

${ }^{2}$ Johnson LV, Hageman GS, Blanks JC: Interphoto-
}

receptor matrix domains ensheath vertebrate cone photoreceptor cells. Invest Ophthalmol Vis Sci 1986, 27: 129-35.

${ }^{3}$ Sameshima M, Uehara F, Ohba, N: Specialization of the interphotoreceptor matrices around cone and rod photoreceptor cells in the monkey retina, as revealed by lectin cytochemistry. Exp Eye Res 1987, 45: 845-63.

${ }^{4}$ Varner HH, Rayborn ME, Osterfeld AM, Hollyfield JG: Localization of proteoglycan within the matrix sheath of cone photoreceptors. Exp Eye Res 1987, 44: 633-42.

${ }^{5}$ Hollyfield, JG, Varner, HH, Rayborn, ME, Osterfeld AM: Retinal attachment to the pigment epithelium: Linkage through an extracellular sheath surrounding cone photoreceptors. Retina 1989, 9: 59-68.

${ }^{6}$ Hageman GS and Johnson LV: Chondroitin 6-sulfate glycosaminoglycan is a major constituent of primate cone photoreceptor matrix sheaths. Curr Eye Res 1987, 6: 639-46.

${ }^{7}$ Johnson, LV and Hageman, GS: Characterization of isolated cone matrix sheath substructure. Invest Ophthalmol Vis Sci (Suppl) 1989, 30: 490.

${ }^{8}$ Hollyfield JG, Rayborn ME, Landers RA: A technique for isolation of the photoreceptor layer from other neurons in the human retina. 1989, Exp Eye Res (In Press).

${ }^{9}$ Scott JE: Proteoglycan histochemistry-a valuable tool for connective tissue biochemists. Collagen Res Rel 1985, 5: 541-75.

${ }^{10}$ Tolentino FI, Schepens CL, Freeman HM: Vitreoretinal Disorders, Diagnosis and Management. Philadelphia: WB Saunders, 1978, 400-412.

${ }^{11}$ Varner HH, Tawara A, Rayborn ME, Osterfeld AM, Landers RA, Hollyfield, JG: The interphotoreceptor matrix of mice and men. Species variations in proteoglycan structure and distribution. Proceedings of the Retina Research Foundation Symposium, The Woodlands, Texas, Portfolio Pub. Co., 1988, 1: 90-107. 